\title{
Determinação da coloração e a disposição de compra pelos consumidores da carne bovina
}

\author{
Rodrigo Augusto Cortez Passetti ${ }^{1 *}$, Juliana Akamine Torrecilhas ${ }^{1}$, Mariana Garcia \\ Ornaghi $^{1}$, Camila Mottin ${ }^{2}$, Ana Guerrero ${ }^{3}$ \\ ${ }^{1}$ Alunos de Pós Graduação - Mestrado - Programa de Pós Graduação em Zootecnia - Universidade \\ Estadual de Maringá. \\ ${ }^{2}$ Aluna de Pós Graduação - Mestrado - Programa de Pós Graduação em Zootecnia - Universidade Estadual de \\ Londrina \\ 3Pós Doutoranda - Bolsista Jovens Talentos - CNPq. - Programa de Pós Graduação em Zootecnia - \\ Universidade Estadual de Maringá. \\ *Autor para correspondência - email:racpassetti@gmail.com
}

\begin{abstract}
RESUMO. A cor desempenha um papel importante na avaliação da qualidade da carne principalmente na hora da decisão da compra. Os consumidores precisam estar primeiro inteiramente satisfeito com as propriedades sensoriais da carne, antes de outras características de qualidade tornam-se relevantes. A metodologia tradicional para avaliação da evolução da cor de carne em exposição é complexa, cara e demanda um longo período para sua realização. O uso de fotografias é uma alternativa para superar essas dificuldades; assim, a validação desse tipo de ferramenta se torna necessário. Um dos maiores problemas nas avaliações de coloração está na elaboração de novas metodologias para obtenção de informações que sejam significativas, que possam comparar e possibilitar a melhora dos produtos. Estas dificuldades podem ser superadas pela a avaliação da cor por meio de fotografias, pois em um sistema de inspeção visual computadorizado; uma imagem e um computador com um software podem realizar diversos objetivos pré-definidos, por isso a sua utilização poderia gerar resultados mais consistentes de maneira eficiente. Assim, nosso objetivo foi revisar a percepção da cor da carne entre a visualização diretamente em um expositor e suas fotografias correspondentes para verificar a confiabilidade e precisão do uso de fotografias de alimentos como ferramenta de avaliação.
\end{abstract}

Palavras chaves: Bovinos, imagens, metodologias, visual

\section{Color determination and consumers acceptance of beef}

\begin{abstract}
Colour perception plays a major role in evaluation of meat quality, especially during purchasing. Consumers need first to be entirely satisfied with the sensory properties of meat, before other quality dimensions become relevant. The traditional methodology of assessing meat color evolution in displays is complex, expensive and demands long duration. Photographs are an alternative to overcome these difficulties thus the validation of this methodology become necessary. One of the biggest problems in coloration assessments is the development of new methodologies to obtain information that is significant; they can compare and improve products. These difficulties can be overcome by assessing the color through photographs, as in a visual inspection system computer an image and a computer with software can perform several pre-defined goals, so its use could lead to more consistent results efficient manner. Thus our objective was to reviser perception of meat between viewing meat directly in displayer and their corresponding photographs to assess the reliability and accuracy of using food photographs as an assessment tool.
\end{abstract}

Keywords: Bovine, images, methodologies, visual 


\section{Considerações iniciais sobre a cor da carne bovina}

A urbanização e aumento da renda são os principais fatores nas mudanças nos padrões de consumo da população. Nas últimas décadas, muitas economias emergentes da Ásia, África e América Latina apresentaram mudanças rápidas e significativas no comportamento do consumo de alimentos (Mazzuchetti, 2004). Raízes, tubérculos e grãos foram gradualmente substituídos por alimentos mais nobres, como trigo, arroz, açúcar, óleos vegetais, produtos lácteos e carnes (Yearbook, 2013). Historicamente, a carne é um produto alimentício bastante apreciado na cultura ocidental, pois é um alimento saboroso e uma excelente fonte de energia, proteínas, minerais e vitaminas (Wood et al., 1999, Webb \& O’Neill, 2008).

Satisfazer os consumidores que demandam produtos de qualidade é o grande desafio da indústria de alimentos. Para esta indústria, a qualidade, é um conjunto de características que diferenciam um produto dos demais que está relacionada à aceitação do produto pelos consumidores (Hocquette et al., 2012, Van Wezemael et al., 2013), os quais levam em consideração vários fatores como seu valor nutritivo, sua conveniência, seu impacto na saúde e suas características sensoriais (Monsón et al., 2005, Hocquette et al., 2011).

A avaliação da qualidade da carne pelo consumidor é baseada na satisfação em experiências passadas e que dependem de um conjunto de respostas psicológicas e sensoriais únicas de cada indivíduo (Ramos \& Gomide, 2007, Fernqvist \& Ekelund, 2014, Verbeke et al., 2010). As características sensoriais dos alimentos são o flavour, a textura e a aparência. O flavour compreende os sentidos do paladar e olfato, a textura compreende o sentido do tato e a aparência (cor, forma, tamanho e brilho) compreende o sentido da visão.

A percepção da cor é um dos fatores mais importantes na avaliação da qualidade da carne pelos consumidores (Lanari et al., 1995, Renerre, 2004, Resconi et al., 2012). Os consumidores precisam estar primeiramente completamente satisfeitos com as propriedades sensoriais da carne antes de qualquer outra característica de qualidade (Chambers \& Bowers, 1993). No caso da carne vermelha, consumidores relacionam a coloração vermelha brilhante com o frescor, enquanto que discriminam carnes que tornaram a cor mais marrom (Hood \& Riordan, 1973, Morrissey et al., 1994, Verbeke et al., 2015).

A aparência irá determinar como os consumidores percebem a qualidade da carne influenciando a sua intenção de compra (Faustman \& Cassens, 1990). No caso da carne bovina, a coloração influencia na intenção de compra mais do que qualquer outro fator (Mancini \& Hunt, 2005), pois é responsável por despertar o desejo de consumir ou rejeitar o produto (Carpenter et al., 2001). Fato o que afeta a aceitação dos consumidores para a carne bovina os quais estão dispostos a pagar mais por carnes que apresentem coloração mais atrativa para eles (Killinger et al., 2004). Embora a coloração visível da superfície da carne mude gradualmente durante os dias de armazenamento.

\section{Cor e visão na espécie humana}

As cores possuíram três dimensões: o matiz é a dimensão que distingue as famílias das cores (vermelho, verde, azul, amarelo), que é determinada pelas diferenças entre os comprimentos de ondas. $\mathrm{O}$ valor é a dimensão que nos permite diferenciar cores mais claras de cores mais escuras. O croma é a dimensão que nos permite observar a intensidade ou grau de saturação das cores(Hunt et al., 1991). Todavia a percepção da cor irá depender da interação entre uma fonte de luz, um objeto iluminado e um detector que para os seres vivos são os olhos e o cérebro.

A luz é um fenômeno físico, ela é composta de partículas de energia que se movem no espaço com propriedades de ondas, os fótons. A aparência de um alimento irá depender de como a luz atinge a sua superfície podendo ser transmitida, refletida ou absorvida. Cada objeto reflete e absorve luz em diferentes porções do espectro. A radiação eletromagnética se estende desde as baixas frequências das ondas de rádio as elevadas frequências dos raios gamas. Entretanto, os olhos dos seres humanos sãos sensíveis a radiação a uma banda estreita de comprimentos denominada de espectro visível que se situa entre 380 a $780 \mathrm{~nm}$ (Ramos \& Gomide, 2007). O diagrama de cromaticidade da CIE representa cada cor fisicamente visível como pontos em um gráfico e pode representar a gama de todas as cores que podem ser produzidas (Lammens, 1994). 


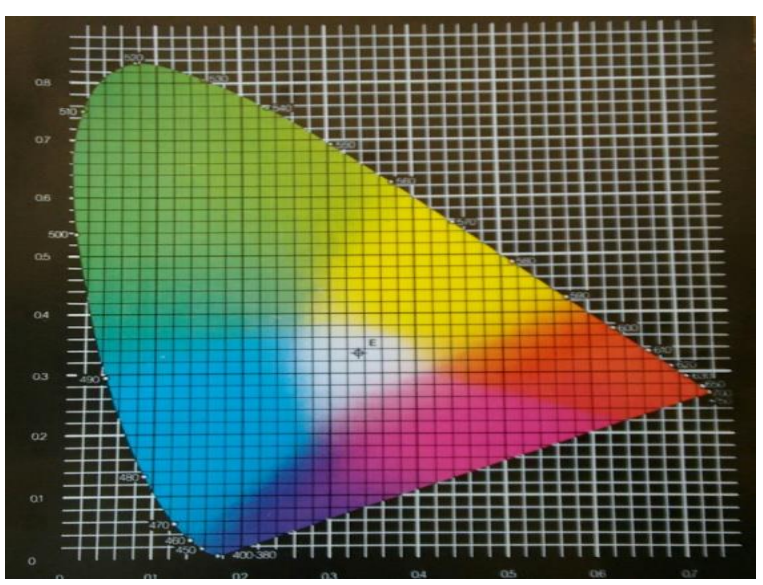

Figura 1.Diagrama de cromaticidade CIE 1931.

Para detectar e processar as diferentes cores, os olhos e o cérebro trabalham sinergicamente. $\mathrm{O}$ olho é composto de duas câmeras que são separadas pela lente, a câmera anterior que é composta da córnea, pupila e íris, e a lente posterior composta pela retina e o nervo óptico (Hunt et al., 1991). O mecanismo da visão se inicia na câmera anterior com a incidência dos raios luminosos refletidos pelo objeto na córnea. Atrás da córnea encontra-se a íris e um orifício chamado de pupila, cujo diâmetro é regulado pela constrição e relaxamento da íris dependendo da intensidade de raios luminosos. Os raios luminosos então serão refratados pela lente ou cristalino e projetados na câmera posterior (Ramos \& Gomide, 2007).

$\mathrm{Na}$ câmera posterior a retina irá captar a luz através de dois tipos de fotorreceptores: os cones e os bastonetes, que chegarão ao cérebro através do nervo óptico. Os bastonetes são sensíveis à intensidade luminosa, enquanto os cones são sensíveis apenas em determinados comprimentos de onda. Os sinais captados pelos cones são conduzidos ao cérebro e interpretados como uma cor específica através das combinações das cores básicas: vermelho, verde e azul. Então cor de um objeto irá depender da sua capacidade de refletir ou emitir diferentes quantidades de energia capazes de estimular a retina do olho (Ramos \& Gomide, 2007).

\section{Coloração da carne}

A mioglobina, o principal pigmento responsável pela coloração da carne bovina, é uma proteína conjugada que consiste de um grupo prostético (grupo heme) ligado a uma molécula de proteína globular (globina). O grupo heme possui um átomo de ferro, podendo ser encontrado tanto na sua forma reduzida (ferrosa,
$\mathrm{Fe}^{2+}$ ) ou oxidada (férrica $\mathrm{Fe}^{3+}$ ). A estrutura do grupo heme e o estado químico do átomo de ferro são os pontos chaves do mecanismo de reações da cor sofridas pelos pigmentos da mioglobina.

A primeira forma química da mioglobina é a deoximioglobina, que ocorre quando nenhum ligante está presente na sexta coordenada de ligação e o ferro do grupo heme esta no estado ferroso $\left(\mathrm{Fe}^{2+}\right)$. Com isso, a mioglobina apresenta uma coloração vermelho-púrpura que é tipicamente associada à carne embalada a vácuo ou de músculo que foi imediatamente cortado. Segundo Brooks (1935), tensões muito baixas de oxigênio (<1.4 milímetros $\mathrm{Hg}$ ) são necessárias para manter a mioglobina em seu estado desoxigenado.

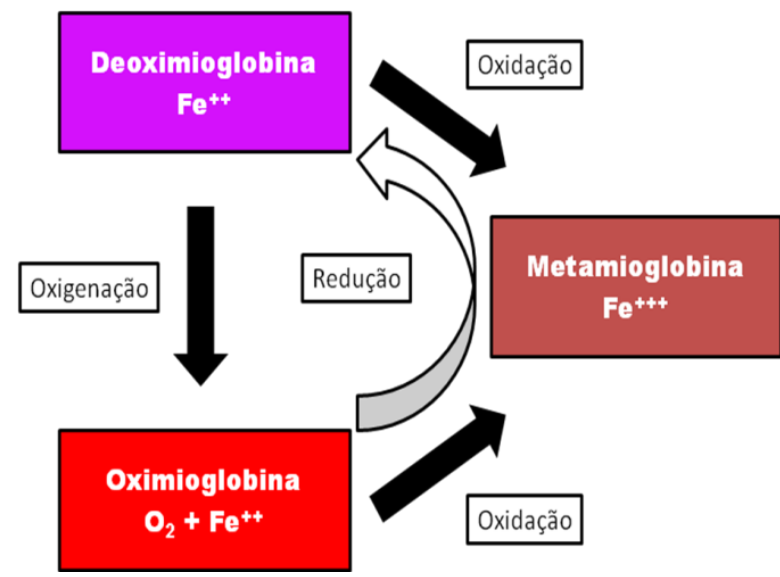

Figura 2. Diagrama da morfologia da mioglobina. Adaptado de (Mancini \& Hunt, 2005).

A segunda forma química é a oximioglobina que ocorre quando a mioglobina é exposta ao oxigênio e é caracterizada pelo desenvolvimento de uma cor vermelho cereja brilhante. Nenhuma mudança na valência do $\mathrm{Fe}^{2+}$ ocorre durante a oxigenação, mas o sítio da coordenada seis é agora ocupado pelo oxigênio. Com o aumento da exposição do oxigênio, a oximioglobina encontrada na superfície penetra mais profundamente na carne. A profundidade da penetração de oxigênio e a espessura da camada da oximioglobina dependeram da temperatura da carne, pressão parcial de oxigênio, pH, e a competição por oxigênio por outros processos respiratórios.A terceira forma química encontrada da mioglobina é a metamioglobina que apresenta uma coloração marrom. A metamioglobina é formada a partir da oxidação de ambos os derivados ferrosos da mioglobina (deoximioglobina e oximioglobina) para ferro 
férrico (Livingston \& Brown, 1981); (Wallace et al., 1982).

A reação de redução da metamioglobina é crucial para a manutenção da coloração da carne a qual irá depender do consumo do oxigênio e do sistema de enzimas redutoras. A redução de oximioglobina superficial da carne é uma reação de duas etapas, pois ela não é convertida diretamente em deoximioglobina, passando primeiro ao estado férrico em condições com baixas concentrações de oxigênio. A remoção endógena de oxigênio acontece pelo seu próprio consumo pelo músculo o que acaba resultando em uma oxidação da oximioglobina para metamioglobina. A formação subsequente da deoximioglobina irá depender da quantidade de enzimas redutoras presentes no músculo capazes de passar o $\mathrm{Fe}^{3+}$ para $\mathrm{Fe}^{2+}$ (Mancini \& Hunt, 2005).

Dentre os três estados da mioglobina, as carnes que apresentaram maior intenção de compra são as de coloração vermelha cereja, depois vermelha púrpura e marrom(Carpenter et al., 2001). Quando a percentagem de metamioglobina atinge 30 a $40 \%$ da superfície da carne a intenção de compra diminui, pois os consumidores associam a cor marrom com carnes de animais velhos ou que foram expostas a venda por muito tempo. (Greene, 1969). Embora a descoloração da carne seja muitas vezes referida como a quantidade de área de superfície coberta por metamioglobina, a superfície da mioglobina também desempenha papel importante na sua aparência. Isso ocorre porque metamioglobina abaixo da superfície localizada entre a oximioglobina superficial e a deoximioglobina interior se engrossa gradualmente e se move para a superfície (Mancini \& Hunt, 2005).

\section{Fatores que afetam a coloração da carne}

A carne é um produto heterogêneo e as diferenças observadas na sua coloração estão envolvidas com inúmeros fatores, sendo os principais responsáveis, o $\mathrm{pH}$ e a quantidade de mioglobina do músculo (Priolo et al., 2001). O $\mathrm{pH}$ final da carcaça está fortemente relacionado com a coloração a luminosidade da carne (Cornforth \& Egbert, 1985). Após a sangria, devido ao cessar da oxigenação e circulação os ATP's são produzidos por via anaeróbica transformando glicogênio em ácido lático. A queda normal do $\mathrm{pH}$ para abaixo de $6,0 \mathrm{em}$ bovinos irá depender da quantidade de glicogênio no músculo.

Em situações de estresse antes do abate os animais consomem a reserva de glicogênio, resultando no surgimento de carnes DFD (Dark, Firm, Dry). Devido ao alto $\mathrm{pH}$ as proteínas conservam uma grande capacidade de reter água no interior das células fazendo com que a superfície do músculo permaneça pegajosa e escura (Maganhini et al., 2007) Além disso a cor da carne também pode ser afetada pela interação de valores altos de $\mathrm{pH}$ e temperatura. Esta combinação de fatores produz uma denaturalização parcial das proteínas miofibrilares e sarcoplasmáticas podendo ocorrer à adsorção da mioglobina a outros elementos protéicos causando um aumento na reflexão da luz resultando em um aspecto pálido da carne característico do PSE (Palid, Soft, Exudative) (Kauffman et al., 1994).

O tecido muscular é composto basicamente por três tipos de fibras musculares: oxidativas de contração lenta (Tipo I, vermelhas e aeróbicas), as glicolíticas de contração rápida (Tipo II A, brancas, anaeróbicas) e as intermediárias de contração rápida (Tipo II $\mathrm{B}$, oxidativas glicolíticas). As fibras vermelhas de contração lenta e metabolismo oxidativo são capazes de melhorar a aceitabilidade da cor da carne, pois aumenta o nível de pigmento vermelho devido a maior quantidade de mioglobina (Henckel et al., 1997). Todavia, a estabilidade da cor está inversamente relacionada ao potencial oxidativo do músculo, principalmente no caso dos bovinos (Renerre, 1984). Fibras musculares que apresentam baixas taxas de consumo de oxigênio permitem que o mesmo penetre mais profundamente proporcionando uma maior estabilidade na cor da carne (McKenna et al., 2005). Por isso um aumento na proporção de fibras predominantemente glicolíticas (brancas) pode apresentar um efeito benéfico sobre a estabilidade da cor da carne, pois apresentam uma menor taxa de consumo de oxigênio (Lefaucheur, 2010).

O tipo de fibra no músculo irá depender do tipo de atividade que este realiza. Quando os músculos são mais exigidos eles apresentam uma maior quantidade de mioglobina, aumentando a proporção de fibras vermelhas entre as fibras brancas (Sañudo et al., 1996). Animais alimentados em sistemas extensivos apresentam a carne mais obscura do que quando confinados, 
pois estes demandam uma maior atividade da musculatura devido ao ato de pastejo ( Muir et al., 1998a; Vestergaard et al., 2000).

A carne é um produto altamente propenso à contaminação de micro-organismos uma vez que ela é rica em nutrientes. A deterioração da carne pelos micro-organismos é acelerada por fatores como o $\mathrm{pH}$ e atividade de água. De maneira geral a carne fresca apresenta atividade de água acima de 0.85 e $\mathrm{pH}$ propício para crescimento de microorganismos (5,0 a 7,0) (Jayasena \& Jo, 2013). As bactérias são os principais micro-organismos que atuam na deterioração da carne decompondo proteínas e lipídios e produzindo características indesejáveis como off-flavours, defeitos na textura e descoloração da carne (Borch et al., 1996).

Segundo Kerry et al. (1998), a incorporação de antioxidantes, às membranas celulares, pode retardar o processo oxidativo da hemoglobina, aumentando o tempo de prateleira. Os antioxidantes atuam na captação dos radicais livres formados pela oxidação lipídica, retardando o processo de conversão do pigmento vermelho da carne (oximioglobina) para o pigmento marrom (metamioglobina) (Hayes et al., 2009). A vitamina E possuí propriedades antioxidantes capazes de reduzir tanto a oxidação lipídica quanto a oxidação da mioglobina quando testadas em carnes embaladas bandejas com papel filme, em embalagens com atmosfera modificada e embalagens a vácuo (Nassu et al., 2012, Buys et al., 2000).

Os óleos essenciais são aditivos orgânicos que possuem atividade antioxidante, pois apresentam compostos fenólicos capazes de interceptar e neutralizar os radicais livres responsáveis pelo processo oxidativo (Hui, 1996). Os extratos de óleos essenciais são metabólitos secundários, podendo ser extraídos de várias partes de uma planta, incluindo folhas, flores, sementes, raízes e cascas (Benchaar et al., 2008). Além disso, os óleos essenciais possuem um efeito direto sobre os micro-organismos responsáveis pela fermentação ruminal, agindo no controle de patógenos pela atividade antimicrobiana, atuando na estrutura da parede celular bacteriana, desnaturando e coagulando as proteínas (Dorman $\&$ Deans, 2000).

O tempo de maturação é muito importante na coloração da carne (Guerrero et al., 2013), pois com o passar do tempo aumenta-se oxidação lipídica, resultando em uma redução da reserva de água nas miofibrilas e consequentemente um aumento na perda de água na carne (HuffLonergan \& Lonergan, 2005) o que ocasiona um aumento da luminosidade. Alguns trabalhos têm mostrado que adição de compostos naturais como própolis não altera a coloração da carne (Zawadzki et al., 2011, Muir et al., 1998b). Todavia dependendo da dosagem utilizada na suplementação de bovinos de corte com óleos essenciais, a intensidade de vermelho ao longo de 14 dias de maturação pode aumentar (Rivaroli et al., 2016). De acordo com estes autores os óleos essenciais poderiam ter diminuído a taxa de consumo de oxigênio, o que resultaria em uma maior quantidade de oxigênio na superfície e uma maior estabilidade da cor da carne (Renerre \& Labas, 1987).

O eugenol e cinamaldeído são os compostos aromáticos mais importantes encontrados nos óleos essenciais de cravo e canela, e são amplamente utilizados na medicina humana, por ser um potente antioxidante, e apresentar propriedades antibacterianas e fungicidas (Hart et al., 2008). Estes óleos quando adicionados diretamente na carne diminuiu consideravelmente o crescimento de bactérias responsáveis pela deterioração da carne (Ouattara et al., 1997).

Embora a utilização de óleos essenciais diretamente na carne aumente a vida de prateleira, dependendo de sua concentração e composto a aceitação pelos consumidores pode diminuir quando avaliados sensorialmente (Chouliara et al., 2007). Por isso, uma alternativa para aumentar vida de prateleira da carne, é o fornecimento destes compostos na dieta dos animais, por ser uma das vias mais eficazes para inibição da oxidação lipídica da gordura animal.

$\mathrm{O}$ uso de antioxidantes, como a vitamina $\mathrm{E}$, na dieta de ruminantes aumenta o tempo de prateleira da carne em até 5 dias sem o comprometimento microbiológico (Gray et al., 1996). Todavia, existem poucos estudos sobre a adição de óleos essenciais na dieta de animais domésticos como também muitas divergências nos seus resultados sobre seu efeito na qualidade de carne. Smeti et al. (2013), ao fornecer 0,06\% de óleo essencial de alecrim na dieta de cordeiros, não observaram diferenças na oxidação lipídica e na descoloração da carne. Entretanto, (Botsoglou et al., 2002) ao fornecer doses diárias de óleo essencial de orégano para frangos de corte observaram uma menor oxidação lipídica. Segundo Rivaroli et al. (2016) 
a suplementação de bovinos terminados em confinamento com 3,5 e 7,0g de um mix de óleos essenciais a base de orégano, alho, limão, alecrim, tomilho, eucalipto e laranja doce observaram uma menor oxidação lipídica na carne de animais alimentados com 3,5g /animal dia do que na carne de animais alimentados com $7,0 \mathrm{~g} /$ animal dia e animais alimentados sem adição óleos essências na dieta.

\section{Métodos de avaliação da cor da carne}

A determinação da cor da carne pode ser feita instrumentalmente com diversos tipos de aparelhos. A natureza e a quantidade de informação que um instrumento de avaliação da cor é capaz dependerão da forma de captação da luz refratada. Os instrumentos podem ser classificados nos que captam apenas um tipo de luz, como os espectofotômetros que são capazes de mensurar o espectro de refletância. Instrumentos que usam três ou quatro tipos de luzes são chamados de colorímetros (Hunt et al., 1991).

Cada instrumento oferece uma gama de opções que variam desde o sistema de coloração (Hunter, CIE, tristimulus) até ao tipo de iluminante (A, C, D65 e Ultralume) (Mancini \& Hunt, 2005). Todavia os mesmos autores afirmam que as coordenadas $\mathrm{L}^{*}, \mathrm{a}^{*} \mathrm{e} \mathrm{b}^{*}$ do sistema de espaço CIELAB é uma das metodologias mais difundidas para determinar a coloração da carne. A coordenada $\mathrm{L}^{*}$ representa a claridade ou luminosidade e é o atributo da percepção visual cujos valores variam de 0 (negro) a 100 (branco). No caso da carne suína o valor de $\mathrm{L}^{*}$ é muito utilizado para predizer carnes pálidas, brancas e exudativas (PSE) (Brewer et al., 2001a). A coordenada $a^{*}$ representa a oposição entre as cores vermelho e verde, sendo os valores positivos correspondentes a cor vermelha e os valores negativos a cor verde, enquanto que a coordenada $b^{*}$ representa a oposição entre as cores azul e amarelo, sendo os valores positivos ao amarelo e os negativo ao azul. A coordenada $a^{*}$ está fortemente correlacionada com o conteúdo de pigmentos hemes e o estado químico da mioglobina já a coordena $b^{*}$ se associa positivamente com a atividade glicolítica e a palidez muscular (Mancini \& Hunt, 2005, Meadus \& MacInnis, 2000).

Entretanto alguns cuidados devem ser tomados ao se comparar resultados entre carcaças, e experimentos, pois o tipo de iluminante ou instrumento pode influenciar na medida da cor. Por isso, para poder fazer comparações, pesquisadores devem padronizar o tempo de blooming, ou seja, o tempo em que a carne é oxigenada (Brewer et al., 2001b).

Além da avaliação da cor por meio de instrumentos, a análise da aceitação visual por consumidores também pode ser realizada. A análise instrumental é mais acurada, rápida e de mais fácil realização, entretanto elas expressam os resultados de cor em coordenadas espaciais que não proporciona uma medida de preferência dos consumidores. A aceitação visual está mais relacionada com a avaliação dos consumidores; entretanto ela é complexa, cara e demanda muito tempo para sua realização (AMSA, 2012).

A percepção da cor é muito dependente do observador, pois é o resultado de um complexo processo de interpretação sensorial, não sendo apenas somente um estímulo e resposta. Por isso, é muito importante saber o valor relativo entre os métodos objetivo de medição da cor com o julgamento subjetivo da aceitação da cor (Verbeke et al., 1999). A análise visual é uma ferramenta muito importante para determinação da aceitação da carne, pois existe uma forte correlação entre cor e intenção de compra, pois os consumidores conseguem descriminar carnes vermelhas de carnes marrons e roxas (Carpenter et al., 2001). Além disso, ao utilizar-se da análise visual consumidores conseguiram detectar diferenças entre lombos suínos com pouca, média e alta quantidade de gordura entremeada, caracterizando os lombos mais marmorizados como mais claros, mais gordos, e com aparência menos aceitável (Brewer et al., 2001b).

O'sullivan et al. (2003), observaram que ao utilizar de um grupo de painel treinados em uma análise visual os avaliadores foram capazes de diferenciar carne de suínos alimentados com diferentes dietas durante os dias de exposição $(0$, 1,3 e 5), como também foram mais efetivos em avaliar a qualidade da cor quando comparados com métodos instrumentais determinados por um colorímetro Minolta. Os mesmos autores afirmaram que o uso da analise visual de produtos cárnicos podem ser realizados de maneira efetiva sem treinamentos, quando os produtos ou a cor dos produtos são familiares aos avaliadores. 


\section{Uso de imagens na avaliação da cor}

Um dos maiores problemas nas avaliações de coloração está na elaboração de novas metodologias para obtenção de informações que sejam significativas, que possam comparar e melhorar os produtos (Girolami et al., 2013). Estas dificuldades podem ser superadas pela a avaliação da cor por meio de fotografias, pois em um sistema de inspeção visual computadorizado uma imagem e um computador com um software podem realizar diversos objetivos pré-definidos, por isso a sua utilização poderia gerar resultados mais consistentes de maneira eficiente ( $\mathrm{Lu}$ et al., 2000).

A utilização de imagens vem se tornando uma alternativa bastante promissora para avaliação da qualidade de carne. Quando comparadas com métodos instrumentais de determinação da cor, O'sullivan et al. (2003) observaram que fotografias apresentaram diversas vantagens como a redução do número de amostras, melhor observação das variações no estado redox da mioglobina superficial e que os resultados poderiam ser convertidos para diferentes sistemas de coloração (Hunter, CIE, XYZ).

Análises com fotografias já foram realizadas com um grupo de painel treinado para predizer a coloração de diferentes lombos suínos (Lu et al., 2000). Estes autores encontraram uma forte correlação entre os escores dos panelistas com os escores calculados por um modelo estatístico. Quando fotografias foram utilizadas com consumidores, estes foram capazes de distinguir colorações de carnes antes e depois do processo de blooming (Brugiapaglia \& Destefanis, 2009). Além disso, consumidores foram capazes de avaliar diferentes graus de cozimento por meio de fotografias da mesma forma quando avaliaram a carne diretamente (Chan et al., 2013).

O método tradicional para avaliação aceitação visual da carne bovina (Prado et al., 2015) possuí algumas dificuldades na sua realização, pois demanda um longo período onde os avaliadores devem comparecer todos os dias para avaliação. Além disso, neste método os avaliadores possuem uma informação adicional sobre o tempo, que na prática os consumidores não possuem na hora da compra. Uma das maiores vantagens da sua utilização com consumidores é a sua repetibilidade, podendo ser usada para avaliar a aceitação de consumidores de diversos lugares no mundo (Ngapo et al., 2004, Ngapo et al., 2007) e em diversos momentos. A utilização de fotografias apresenta ser uma alternativa promissora, por essa razão, como primeiro passo, torna-se necessário a validação da sua utilização na redução dos efeitos causados pelas diferenças observadas entre consumidores para sua utilização como método alternativo a avaliação direta sobre o produto.

\section{Conclusões}

A coloração da carne bovina é determinante na hora da compra do produto. Sua mensuração por ser realizada de forma direta: comprador julgamento do comprador e indireta mensurações instrumentais. No entanto, na atualidade se busca ferramentas que sejam eficiente e mais barata na avaliação da cor a carne. Desta forma, o uso de fotografias padronizadas pode ser uma ferramenta útil na avaliação da cor da carne. Todavia, esta metodologia deve ser usada por especialistas treinados para essa finalidade.

\section{Referências Bibliográficas}

AMSA. (2012). Meat color measurement guidelines. In: Association, A. M. S. (ed.). National Livestock and Meat Board, Savoy, IL.

Benchaar, C., Calsamiglia, S., Chaves, A. V., Fraser, G. R., Colombatto, D., McAllister, T. A. \& Beauchemin, K. A. (2008). A review of plant-derived essential oils in ruminant nutrition and production. Animal Feed Science and Technology, 145, 209-228.

Borch, E., Kant-Muermans, M.-L. \& Blixt, Y. (1996). Bacterial spoilage of meat and cured meat products. International Journal of Food Microbiology, 33, 103-120.

Botsoglou, N., Christaki, E., Fletouris, D., Florou-Paneri, P. \& Spais, A. (2002). The effect of dietary oregano essential oil on lipid oxidation in raw and cooked chicken during refrigerated storage. Meat Science, 62, 259265.

Brewer, M., Zhu, L., Bidner, B., Meisinger, D. \& McKeith, F. (2001a). Measuring pork color: effects of bloom time, muscle, $\mathrm{pH}$ and relationship to instrumental parameters. Meat Science, 57, 169-176.

Brewer, M., Zhu, L. \& McKeith, F. (2001b). Marbling effects on quality characteristics of pork loin chops: consumer purchase intent, 
visual and sensory characteristics. Meat Science, 59, 153-163.

Brooks, J. (1935). The oxidation of haemoglobin to methaemoglobin by oxygen. II.-The relation between the rate of oxidation and the partial pressure of oxygen. Proceedings of the Royal Society of London. Series B, Biological Sciences, 118, 560-577.

Brugiapaglia, A. \& Destefanis, G. (2009). Sensory evaluation of meat colour using photographs. Italian Journal of Animal Science, 8, 480-482.

Buys, E. M., Nortjé, G. L., Jooste, P. J. \& Von Holy, A. (2000). Combined effect of modified atmosphere bulk packaging, dietary vitamin $\mathrm{E}$ supplementation and microbiological contamination on colour stability of Musculus gluteus medius. Meat Science, 55, 403-411.

Carpenter, C. E., Cornforth, D. P. \& Whittier, D. (2001). Consumer preferences for beef color and packaging did not affect eating satisfaction. Meat Science, 57, 359-363.

Chambers, E. \& Bowers, J. (1993). Consumer perception of sensory qualities in muscle foods. Food technology (USA).

Chan, S. H., Moss, B. W., Farmer, L. J., Gordon, A. \& Cuskelly, G. J. (2013). Comparison of consumer perception and acceptability for steaks cooked to different endpoints: Validation of photographic approach. Food Chemistry, 136, 1597-1602.

Chouliara, E., Karatapanis, A., Savvaidis, I. N. \& Kontominas, M. G. (2007). Combined effect of oregano essential oil and modified atmosphere packaging on shelf-life extension of fresh chicken breast meat, stored at $4 \mathrm{C}$. Food Microbiology, 24, 607-617.

Cornforth, D. P. \& Egbert, W. R. (1985). Effect of rotenone and $\mathrm{pH}$ on the color of pre-rigor muscle. Journal of Food Science, 50, 34-35.

Dorman, H. J. D. \& Deans, S. G. (2000). Antimicrobial agents from plants: antibacterial activity of plant volatile oils. Journal of Applied Microbiology, 88, 308316.

Faustman, C. \& Cassens, R. G. (1990). The biochemical basis for discoloration in fresh meat: a review. Journal of Muscle Foods, 1, 217-243.
Fernqvist, F. \& Ekelund, L. (2014). Credence and the effect on consumer liking of food - A review. Food Quality and Preference, 32, 340-353.

Girolami, A., Napolitano, F., Faraone, D. \& Braghieri, A. (2013). Measurement of meat color using a computer vision system. Meat Science, 93, 111-118.

Gray, J., Gomaa, E. \& Buckley, D. (1996). Oxidative quality and shelf life of meats. Meat Science, 43, 111-123.

Greene, B. E. (1969). Lipid oxidation and pigment changes in raw beef. Journal of Food Science, 34, 110-113.

Guerrero, A., Sañudo, C., Albertí, P., Ripoll, G., Campo, M. M., Olleta, J. L., Panea, B., Khliji, S. \& Santolaria, P. (2013). Effect of production system before the finishing period on carcass, meat and fat qualities of beef. Animal, 7, 2063-2072.

Hart, K. J., Yáñez-Ruiz, D. R., Duval, S. M., McEwan, N. R. \& Newbold, C. J. (2008). Plant extracts to manipulate rumen fermentation. Animal Feed Science and Technology, 147, 8-35.

Hayes, J., Stepanyan, V., Allen, P., O’Grady, M., O'Brien, N. \& Kerry, J. (2009). The effect of lutein, sesamol, ellagic acid and olive leaf extract on lipid oxidation and oxymyoglobin oxidation in bovine and porcine muscle model systems. Meat Science, 83, 201-208.

Henckel, P., Oksbjerg, N., Erlandsen, E., BartonGade, P. \& Bejerholm, C. (1997). Histo-and biochemical characteristics of the longissimus dorsi muscle in pigs and their relationships to performance and meat quality. Meat Science, 47, 311-321.

Hocquette, J.-F., Botreau, R., Picard, B., Jacquet, A., Pethick, D. W. \& Scollan, N. D. (2012). Opportunities for predicting and manipulating beef quality. Meat Science, 92, 197-209.

Hocquette, J. F., Legrand, I., Jurie, C., Pethick, D. W. \& Micol, D. (2011). Perception in France of the Australian system for the prediction of beef quality (Meat Standards Australia) with perspectives for the European beef sector. Animal Production Science, 51, 30-36.

Hood, D. E. \& Riordan, E. B. (1973). Discolouration in pre-packaged beef: 
Measurement

spectrophotometry

by and

reflectance discrimination. International Journal of Food Science \& Technology, 8, 333-343.

Huff-Lonergan, E. \& Lonergan, S. M. (2005). Mechanisms of water-holding capacity of meat: The role of postmortem biochemical and structural changes. Meat Science, 71, 194204.

Hui, Y. H. (1996). Oleoresins and essential oils. Bailey's industrial oil and fat products. New York: Wiley-Interscience Publication, 6, 145153.

Hunt, M., Acton, J., Benedict, R., Calkins, C., Cornforth, D., Jeremiah, L., Olson, D., Salm, C., Savell, J. \& Shivas, S. (1991). Guidelines for meat color evaluation. American Meat Science Association.

Jayasena, D. D. \& Jo, C. (2013). Essential oils as potential antimicrobial agents in meat and meat products: A review. Trends in Food Science \& Technology, 34, 96-108.

Kauffman, R. G., Marsh, B. B., Price, J. F. \& Schweigert, B. S. (1994). Características de calidad del músculo como alimento. Ciencia de la Carne y de los Productos Carnicos, 2, 317-336.

Kerry, J., Buckley, D., Morrissey, P., O'Sullivan, K. \& Lynch, P. (1998). Endogenous and exogenous $\alpha$-tocopherol supplementation: effects on lipid stability (TBARS) and warmed-over flavour (WOF) in porcine $\mathrm{M}$. longissimus dorsi roasts held in aerobic and vacuum packs. Food Research International, 31, 211-216.

Killinger, K., Calkins, C. R., Umberger, W., Feuz, D. M. \& Eskridge, K. M. (2004). Consumer visual preference and value for beef steaks differing in marbling level and color. Journal of Animal Science, 82, 32883293.

Lammens, J. M. G. (1994). A computational model of color perception and color naming. Faculty of the Graduate School. State University of New York, New York.

Lanari, M. C., Schaefer, D. M., Cassens, R. G. \& Scheller, K. K. (1995). Atmosphere and blooming time affect color and lipid stability of frozen beef from steers supplemented with Vitamin E. Meat Science, 40, 33-44.
Lefaucheur, L. (2010). A second look into fibre typing - Relation to meat quality. Meat Science, 84, 257-270.

Livingston, D. J. \& Brown, W. D. (1981). The chemistry of myoglobin and its reactions [Meat pigments, food quality indices]. Food Technology.

Lu, J., Tan, J., Shatadal, P. \& Gerrard, D. E. (2000). Evaluation of pork color by using computer vision. Meat Science, 56, 57-60.

Maganhini, M. B., Mariano, B., Soares, A. L., Guarnieri, P. D., Shimokomaki, M. \& Ida, E. I. (2007). Carnes PSE (Pale, Soft, Exudative) e DFD (Dark, Firm, Dry) em lombo suíno numa linha de abate industrial. Ciência $e$ Tecnologia de Alimentos, 27, 69-72.

Mancini, R. A. \& Hunt, M. C. (2005). Current research in meat color. Meat Science, 71, 100121.

Mazzuchetti, R. N. (2004). O comportamento do consumidor em relação ao consumo e às estruturas de comercialização da carne bovina na região de Amerios/PR. Varia Scientia, 4, 923.

McKenna, D. R., Mies, P. D., Baird, B. E., Pfeiffer, K. D., Ellebracht, J. W. \& Savell, J. W. (2005). Biochemical and physical factors affecting discoloration characteristics of 19 bovine muscles. Meat Science, 70, 665-682.

Meadus, W. J. \& MacInnis, R. (2000). Testing for the RN- gene in retail pork chops. Meat Science, 54, 231-237.

Monsón, F., Sañudo, C. \& Sierra, I. (2005). Influence of breed and ageing time on the sensory meat quality and consumer acceptability in intensively reared beef. Meat Science, 71, 471-479.

Morrissey, P. A., Buckley, D. J., Sheehy, P. J. A. \& Monahan, F. J. (1994). Vitamin E and meat quality. Proceedings of the Nutrition Society, 53, 289-295.

Muir, P., Smith, N., Wallace, G., Cruickshank, G. \& Smith, D. (1998a). The effect of short-term grain feeding on liveweight gain and beef quality. New Zealand Journal of Agricultural Research, 41, 517-526.

Muir, P. D., Deaker, J. M. \& Bown, M. D. (1998b). Effects of forage-and grain-based feeding systems on beef quality: A review. 


\section{New Zealand Journal of Agricultural} Research, 41, 623-635.

Nassu, R. T., Uttaro, B., Aalhus, J. L., Zawadski, S., Juárez, M. \& Dugan, M. E. R. (2012). Type of packaging affects the colour stability of vitamin E enriched beef. Food Chemistry, 135, 1868-1872.

Ngapo, T. M., Martin, J. F. \& Dransfield, E. (2004). Consumer choices of pork chops: results from three panels in France. Food Quality and Preference, 15, 349-359.

Ngapo, T. M., Martin, J. F. \& Dransfield, E. (2007). International preferences for pork appearance: II. Factors influencing consumer choice. Food Quality and Preference, 18, 139-151.

O'sullivan, M. G., Byrne, D. V., Martens, H., Gidskehaug, L. H., Andersen, H. J. \& Martens, M. (2003). Evaluation of pork colour: prediction of visual sensory quality of meat from instrumental and computer vision methods of colour analysis. Meat Science, 65, 909-918.

Ouattara, B., Simard, R. E., Holley, R. A., Piette, G. J. P. \& Bégin, A. (1997). Antibacterial activity of selected fatty acids and essential oils against six meat spoilage organisms. International Journal of Food Microbiology, 37, 155-162.

Prado, I. N., Campo, M. M., Muela, E., Valero, M. V., Catalan, O., Olleta, J. L. \& Sañudo, C. (2015). Effects of castration age, protein level and lysine/methionine ratio in the diet on colour, lipid oxidation, and meat cceptability of intensively reared Friesian steers. Animal, 9, 1423-1430.

Priolo, A., Micol, D. \& Agabriel, J. (2001). Effects of grass feeding systems on ruminant meat colour and flavour. A review. Animal Research, 50, 185-200.

Ramos, E. M. \& Gomide, L. A. M. (2007). Avaliação da qualidade de carnes: fundamento $e$ metodologias. Universidade Federal de Viçosa,Viçosa.

Renerre, M. (1984). Variabilité entre muscles et entre animaux de la stabilité de la couleur des viandes bovines. Sciences des Aliments.

Renerre, M. (2004). La couleur de la viande et sa mesure. INRA, 1, 63-68.
Renerre, M. \& Labas, R. (1987). Biochemical factors influencing metmyoglobin formation in beef muscles. Meat Science, 19, 151-165.

Resconi, V. C., Escudero, A., Beltrán, J. A., Olleta, J. L., Sañudo, C. \& Campo, M. M. (2012). Color, lipid oxidation, sensory quality, and aroma compounds of beef steaks displayed under different levels of oxygen in a modified atmosphere package. Journal of Food Science, 71, S10-S18.

Rivaroli, D. C., Guerrero, A., Valero, M. M., Zawadzki, F., Eiras, C. E., Campo, M. M., Sañudo, C., Jorge, A. M. \& Prado, I. N. (2016). Effect of essential oils on meat and fat qualities of crossbred young bulls finished in feedlot. Meat Science, in press.

Sañudo, C., Santolaria, M. P., María, G., Osório, M. T. \& Sierra, I. (1996). Influence of carcass weight on instrumental and sensory lamb meat quality in intensive production systems. Meat Science, 42, 195-202.

Smeti, S., Atti, N., Mahouachi, M. \& Munoz, F. (2013). Use of dietary rosemary (Rosmarinus officinalis L.) essential oils to increase the shelf life of Barbarine light lamb meat. Small Ruminant Research, 113, 340-345.

Van Wezemael, L., De Smet, S., Ueland, O. \& Verbeke, W. (2013). Relationships between sensory evaluations of beef tenderness, shear force measurements and consumer characteristics. Meat Science.

Verbeke, W., Marcu, A., Rutsaert, P., Gaspar, R., Seibt, B., Fletcher, D. \& Barnett, J. (2015). Would you eat cultured meat?: Consumers' reactions and attitude formation in Belgium, Portugal and the United Kingdom. Meat Science, 102, 49-58.

Verbeke, W., Van Oeckel, M. J., Warnants, N., Viaene, J. \& Boucque, C. V. (1999). Consumer perception, facts and possibilities to improve acceptability of health and sensory characteristics of pork. Meat science, 53, 7799.

Verbeke, W., Van Wezemael, L., Barcellos, M. D., Kügler, J. O., Hocquette, J. F., Ueland, Ø. \& Grunert, K. G. (2010). European beef consumers' interest in a beef eating-quality guarantee. Insights from a qualitative study in four EU countries. Appetite, 54, 289-296.

Vestergaard, M., Oksbjerg, N. \& Henckel, P. (2000). Influence of feeding intensity, grazing 
and finishing feeding on muscle fibre characteristics and meat colour of (semitendinosus, longissimusdorsi) and (supraspinatus) muscles of young bulls. Meat Science, 54, 177-185.

Wallace, W. J., Houtchens, R. A., Maxwell, J. C. \& Caughey, W. S. (1982). Mechanism of autooxidation for hemoglobins and myoglobins. Promotion of superoxide production by protons and anions. Journal of Biological Chemistry, 257, 4966-4977.

Webb, E. C. \& O'Neill, H. A. (2008). The animal fat paradox and meat quality. Meat Science, 80, 28-36.

Wood, J. D., Enser, M., Fisher, A. V., Nute, G. R., Richardson, R. I. \& Sheard, P. R. (1999). Manipulating meat quality and composition. Proceedings of the Nutrition Society, 58, 363370.
Yearbook, F. S. (2013). World food and agriculture. Food and Agriculture Organization of the United Nations, Rome.

Zawadzki, F., Prado, I. N., Marques, J. A., Zeoula, L. M., Rotta, P. P., Sestari, B. B., Valero, M. V. \& Rivaroli, D. C. (2011). Sodium monensin or propolis extract in the diets of feedlot-finished bulls: effects on animal performance and carcass characteristics. Journal of Animal and Feed Sciences, 20, 16-25.

Recebido emDezembro 11, 2015

Aceito em Janeiro 6, 2016

License information: This is an open-access article distributed under the terms of the Creative Commons Attribution License, which permits unrestricted use, distribution, and reproduction in any medium, provided the original work is properly cited. 